A. Holstege

\section{Das akute Leberversagen}

\section{Acute liver failure}

Summary Acute liver failure represents a serious life-threatening event comparable to acute heart failure with cardiogenic shock or acute renal failure. Underlying acute liver diseases leading to hepatic failure differ between different geographic regions and in their incidence rates. In Europe etiological agents like viruses, drugs and toxins predominate over other much rarer causes. The different noxious agents lead to hepatocellular necrosis and/or apoptosis with loss of liver cell specific functions subsequent to a fall of functioning hepatocytes below a critical number. The syndrome is clinically characterized by the rapid

Eingegangen: 15. November 2002

Akzeptiert: 17. Januar 2003

\section{Serie:}

Die Intensivtherapie

in der Gastroenterologie

Herausgegeben von

J. Schölmerich (Regensburg)

Prof. Dr. Axel Holstege (

Klinikum Landshut

Medizinische Klinik I

Robert-Koch-Straße 1

84028 Landshut, Germany

Tel.: 08 71/698-37 17

Fax: 0871/698-3476 onset of hepatic encephalopathy within 7 days after a first manifestation of liver disease (fulminant liver disease). Liver failure in patients with preexisting chronic liver disease is largely defined by the time which elapses between the occurrence of jaundice and encephalopathy (hyperacute, acute, subacute liver failure). The acute loss of liver specific functions is accompanied by a number of severe life-threatening complications like cerebral edema, circulatory failure, infections, renal failure and defective coagulation. Management of patients with fulminant liver disease requires a profound knowledge of hepatology and intensive care medicine. A close cooperation with a liver transplant unit is an absolute prerequisite for successful therapy. Permanent or temporary auxiliary liver replacement by a healthy human liver allows for a survival of 60 to $70 \%$ of patients selected for such a transplant procedure. Progress has been made in the temporary substitution of specific liver cell functions bridging the time period between liver failure and resumption of hepatocellular functions or availability of a donor liver. Different artificial livers have been designed and introduced into clinical trials. However, further evaluation is urgently needed.
Key words Acute liver failure liver disease - therapy liver assist device

Zusammenfassung Das akute Leberversagen stellt einen seltenen Notfall vergleichbar der akuten Herzinsuffizienz mit kardiogenem Schock oder dem akuten Nierenversagen dar. Die Ursachen für ein akutes Leberversagen sind je nach geographischer Region verschieden und variieren in ihrer Häufigkeit. In unseren Breiten finden sich häufig Viren, Medikamente und Toxine als ätiologisches Agens. Die Einwirkung der Noxe führt zur Nekrose und/oder gesteigerten Apoptose der Hepatozyten mit Ausfall leberspezifischer Funktionen, sobald die Zahl funktionstüchtiger Zellen eine kritische Grenze unterschreitet. Klinisch äußert sich dies Syndrom durch das rasche Auftreten einer hepatischen Encephalopathie innerhalb von 7 Tagen nach Beginn der Lebererkrankung (fulminantes Leberversagen). Bei vorbestehender chronischer Leberschädigung definiert sich das Leberversagen über die Zeit, innerhalb der eine hepatische Encephalopathie nach Beginn eines Ikterus auftritt (hyperakutes, akutes und subakutes Leberversagen). Der Ausfall der Leberfunktion löst zahlreiche extrahepatische lebensbedrohliche Komplikationen 
aus, wie Hirnödem, Kreislaufversagen, Infektionen, Gerinnungsstörungen und Nierenversagen. Intensivmedizinische und hepatologische Kenntnisse und eine enge Kooperation mit einem Lebertransplantationszentrum sind wesentliche Voraussetzungen für eine erfolgreiche Therapie. Der permanente oder auxiliäre vorübergehende Leberersatz durch
Implantation einer gesunden menschlichen Leber ermöglicht in $60-70 \%$ der Fälle ein dauerhaftes Überleben. Fortschritte wurden durch den temporären extrakorporalen Ersatz bestimmter Leberfunktionen erreicht. Hierdurch kann möglicherweise zukünftig die Zeit bis zur Regeneration der eigenen Leber oder bis eine Spenderleber zur Verfügung steht überbrückt werden. Diese Systeme sind für einen Einsatz in der täglichen Routine jedoch noch nicht ausreichend evaluiert.

\section{Schlüsselwörter}

Akutes Leberversagen Leberkrankheiten- Therapie Künstliche Leber
Der Ablauf des fulminanten Leberversagens lässt sich zeitlich in verschiedene Abschnitte gliedern (1), die unterschiedliche therapeutische Ansätze ermöglichen (Abb. 1). Durch die Einwirkung der schädigenden Noxe kommt es zum Leberzerfall mit Nekrose und/ oder rasch ablaufender Apoptose der Hepatozyten. Das Organversagen tritt auf, wenn die Zahl der noch funktionstüchtigen Leberzellen so gering wird, dass die leberspezifischen Funktionen nicht mehr aufrechterhalten werden können. Die Prognose des Patienten wird somit von der noch vorhandenen funktionstüchtigen Leberzellmasse und von der Dauer der Regenerationszeit, die zur Restitutio ad integrum erforderlich ist, bestimmt. Das schädigende Agens selbst und möglicherweise Mediatoren, die von geschädigten Leberzellen oder Kupffer-Zellen abgegeben werden, führen nicht nur zur Freisetzung von proregeneratorischen hepatotrophen Faktoren, sondern auch von deren Antagonisten.

Schwierigkeiten bereitet das Erkennen des Syndroms des akuten Leberversagens dadurch, dass der Schweregrad der Leberschädigung nicht immer mit einem entsprechenden Anstieg der Transaminasen im Serum einhergeht (2) und die Klärung der Ursache mittels serologischer Diagnostik (3) in dieser Situation versagen kann.

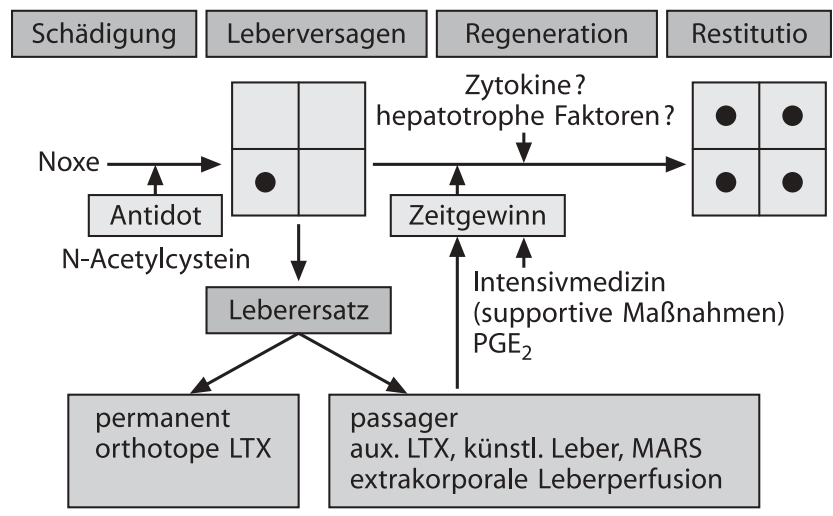

Abb. 1 Therapieoptionen im zeitlichen Ablauf des Leberversagens
Selten kann die Wirkung der Noxe auf die Leberzelle durch frühzeitige Gabe eines entsprechenden Antidots verhindert werden. Dies ist z. B. durch die rechtzeitige Gabe von N-Acetylcystein bei einer $\mathrm{Pa}$ racetamolvergiftung möglich. Ähnliche kausale Therapieansätze sind bei der Knollenblätterpilzvergiftung durch Hemmung der Giftresorption oder bei einer Herpes simplex- oder Zytomegalie-Virus-Infektion durch die Gabe von Virustatika möglich.

Im Stadium des Leberausfalls ist die schwierige therapeutische Entscheidung $(1,47)$ zwischen einem konservativen Vorgehen oder einem vollständigen Leberersatz zu treffen (Abb. 1). Verschiedene prognostische Parameter, die eine Aussage zur Überlebenswahrscheinlichkeit des Patienten erlauben, sind für diese Entscheidung hilfreich. Bei zu weit fortgeschrittenem Verlust der Leberfunktion muss entweder ein permanenter oder passagerer Leberersatz (intra- oder extrakorporal) erfolgen. Bei der orthotopen Lebertransplantation wird die geschädigte Leber des Patienten durch eine gesunde Spenderleber ersetzt. Ein passagerer Leberersatz, z.B. durch eine auxiliäre Lebertransplantation, dient dazu, die Funktion der weitgehend nekrotisch gewordenen Leber vorübergehend zu übernehmen und somit Zeit für die Regeneration der eigenen Leber zu gewinnen. Dieser Funktion dient auch der extrakorporale Leberersatz durch eine „künstliche Leber". In der Phase der Regeneration dienen sämtliche medizinischen konservativen Maßnahmen dazu, die vitalen Funktionen der extrahepatischen Organe aufrechtzuerhalten und ein Multiorganversagen $\mathrm{zu}$ verhindern. Unter diesen Bedingungen kann ebenfalls eine vollständige Restitutio ad integrum erreicht werden.

Bei Patienten mit akutem Leberversagen wird entsprechend das weitere therapeutische Vorgehen von folgenden Faktoren bestimmt:

1. der Ätiologie der Lebererkrankung,

2. dem Ausmaß der extrahepatischen Komplikationen,

3. dem Vorliegen von Kontraindikationen gegen eine Lebertransplantation, 
4. der Einschätzung der Prognose anhand geeigneter Parameter.

\section{Definition}

Lange Zeit verstand man unter dem fulminanten Leberversagen das Auftreten einer schweren Leberfunktionsstörung zusammen mit einer hepatischen Enzephalopathie innerhalb von 8 Wochen nach Beginn der Lebererkrankung (4). Diese Definition schloss Patienten mit vorbestehender Lebererkrankung aus. Nach einer neueren Definition (5) wird unter einem hyperakuten Leberversagen das Auftreten einer hepatischen Enzephalopathie innerhalb von 7 Tagen nach Beginn eines Ikterus verstanden. Das akute Leberversagen ist durch das Auftreten einer hepatischen Enzephalopathie innerhalb von 8-28 Tagen nach Beginn des Ikterus definiert. Von einem subakutem Leberversagen wird gesprochen, wenn sich die hepatische Enzephalopathie 5-12 Wochen nach Auftreten des Ikterus erstmals manifestiert.

Die so charakterisierten Patientengruppen unterscheiden sich im Hinblick auf die Inzidenz des zerebralen Ödems und die Prognose (6). Patienten mit subakutem Leberversagen erleiden seltener ein zerebrales Ödem, haben aber dennoch eine schlechtere Prognose als Patienten mit hyperakutem Leberversagen. Während diese Patienten gut auf eine konservative Therapie ansprechen, benötigen Patienten mit akutem Leberversagen fast immer eine Lebertransplantation. Diese Definitionen des Leberversagens sind hilfreich aber nicht unumstritten (7).

\section{Ätiologie des akuten Leberversagens}

Die Ursachen für ein akutes Leberversagen sind vielfältig (Tab. 1) und je nach geographischer Region verschieden (8). Eine virale Genese findet sich definitiv in $40-50 \%$ der in Europa eingewiesenen Fälle, wobei in weiteren $17 \%$ der Fälle eine solche Genese vermutet wird (6). Der Nachweis der verursachenden Viren gelingt gerade beim fulminanten Leberversagen nicht immer und bedarf gegebenenfalls wiederholter serologischer und molekularbiologischer Bestimmungen $(3,9)$. Nicht selten ist mehr als ein hepatotrophes Virus zu finden (9).

Ein akutes Leberversagen tritt nur in etwa 0,3\% der Fälle mit akuter Hepatitis A auf und betrifft ausschließlich Erwachsene, die die Infektion zumeist im Rahmen von touristischen Aktivitäten erworben haben (10). Die Diagnose wird durch den Nachweis von IgM-Antikörpern gegen das Hepatitis-A-Virus
Tab. 1 Ursachen für ein akutes Leberversagen

\begin{tabular}{ll}
\hline Einteilung & Spezifische Ätiologie \\
\hline Infektionen & $\begin{array}{l}\text { Hepatitis-Virus A, B, C, D, E (allein oder in } \\
\text { Kombination) Cytomegalievirus, Epstein-Barr-Virus, } \\
\text { Herpes simplex virus, Paramyxovirus (Riesenzell- } \\
\text { hepatitis) }\end{array}$ \\
Stoffwechselstörung & $\begin{array}{l}\text { Akute Schwangerschaftsfettleber, Leberversagen } \\
\text { nach jejunoilealem Bypass, Reyes-Syndrom, }\end{array}$ \\
& $\begin{array}{l}\text { Morbus Wilson, Erythropoetische Protoporphyrie } \\
\text { Medikamente/Toxine }\end{array}$ \\
& $\begin{array}{l}\text { Paracetamol, Tetracyclin, Halothan, Isoniazid } \\
\text { (vor allem Kombination mit Pyrazinamid), }\end{array}$ \\
& $\begin{array}{l}\text { Rifampicin, NSAR, Fialuridin, Methyldopa, } \\
\text { Valproinsäure, etc. } \\
\text { Tetrachlorkohlenstoff, gelber Phosphor, Knollen- } \\
\text { blätterpilz, Toxin von Bacillus cereus (Nahrungs- } \\
\text { mittel), Ecstasy, Mikrocystin aus Cyanobakterien } \\
\text { (kontaminiertes Wasser bei Hämodialyse), } \\
\text { Kava-Kava } \\
\text { Lebervenenverschluss (Budd-Chiari), Leber- } \\
\text { arterienverschluss, Schock, Hyperthermie, } \\
\text { primäres Transplantatversagen nach Lebertrans- } \\
\text { plantation, Leberversagen nach TIPS-Implantation, } \\
\text { Tumorinfiltration }\end{array}$ \\
\hline
\end{tabular}

im Serum gestellt. Etwa 40\% dieser Patienten überstehen die Erkrankung ohne Lebertransplantation (11).

1-3\% der Hepatitis-B-Virus-Infektionen (HBV) führen $\mathrm{zu}$ einem akuten Leberversagen. Von diesen Patienten benötigen $80 \%$ eine Lebertransplantation. Die gleichzeitige Infektion dieses Virus mit dem Hepatitis-D-Virus (HDV) führt seltener zu einer fulminanten Verlauf (Co-Infektion) als die HDV-Infektion bei vorbestehender chronischer Hepatitis B (Superinfektion). Die Diagnose der HBV-Infektion wird durch den Nachweis des HbsAg im Serum und/oder von IgM-Antikörpern gegen das Core-Antigen (AntiHBc-IgM) im Serum gestellt. Die Bestimmung der HBV-DNA bei negativer Serologie ermöglicht in sehr seltenen Fällen die Identifikation von weiteren Patienten mit HBV-Infektion $(3,12)$. Bei der Delta-Virus-Hepatitis ist der Nachweis von Anti-HDV-IgM erforderlich. In einigen Fällen (14\%, 2/14) konnte die HDV-Infektion ausschließlich über die Bestimmung der HDVRNA gesichert werden. Mutationen im Bereich der Präcore-Region der Hepatitis-B-Virus-DNA führen wahrscheinlich häufiger zu einem fulminanten oder schweren Verlauf der Hepatitis $(13,14)$.

Das Hepatitis-C-Virus ist im Gegensatz zum asiatischen Kontinent in westlichen Ländern selten für ein fulminantes Leberversagen verantwortlich $(9,11$, 15). Allerdings führte eine zusätzliche Infektion mit dem Hepatitis-A-Virus bei Patienten mit chronischer Hepatitis $\mathrm{C}$ bei fast $40 \% \mathrm{zu}$ einem fulminanten Verlauf (16). Auch kann das Absetzen einer Chemotherapie wegen eines Malignoms bei HCV-Infizierten $\mathrm{zu}$ 
einer fulminanten Hepatitis führen (17). Der Nachweis der HCV-Infektion erfolgt über die Bestimmung von Anti-HCV und beim akuten Leberversagen vorzugsweise über die HCV-RNA im Serum.

Die Hepatitis $\mathrm{E}$ ist insbesondere in Endemiegebieten bei schwangeren Frauen eine häufigere Ursache (ca. 10\%) für ein akutes Leberversagen $(18,19)$. In unseren Breiten kommt diese Erkrankung nur bei Touristen aus diesen Ländern (Südostasien, Indien, Mittelamerika) vor. Die Bestimmung von Antikörpern oder der HEV-RNA muss in hierfür eingerichteten Labors erfolgen. Unklar ist die Bedeutung von HEV-Infektionen bei fulminantem Leberversagen außerhalb von Endemiegebieten (20).

Die Erstmanifestation eines Morbus Wilson über ein akutes Leberversagen ist selten (2). Das Absetzen der lebenslang durchzuführenden Therapie mit Penicillamin ist mit dem Auftreten eines akuten Leberversagens assoziert. Als sicherster Parameter zur Erkennung eines fulminant verlaufenden Morbus Wilson erwies sich eine gesteigerte Kupferausscheidung im Urin. Die Konzentration von Kupfer im Serum und in der Leber sowie das Coeruloplasmin war bei einem Teil der 21 Patienten in einer Studie normal (2).

Schädigungen der Leber durch Medikamente, Toxine, ausgedehnte Lebermetastasierung oder Tumorinfiltration (21), eine akute Leberischämie oder die akute Schwangerschaftsfettleber sind weitere Ursachen für das akute Leberversagen. In England und den USA ist die Paracetamolintoxikation die mit $52 \%$ häufigste Ursache für ein Leberversagen (11). Das Medikament wird häufig in suizidaler Absicht eingenommen und induziert auch in geringeren Dosen bei alkoholisch vorgeschädigter Leber einen ausgedehnten Leberzerfall $(22,23)$. Ein thrombotischer Verschluss aller 3 Lebervenen kann bei entsprechend prädisponierten Patienten (Protein S-Mangel, Protein C-Mangel, etc.) zum akuten Budd-Chiari-Syndrom mit fulminantem Leberversagen führen. Auch an pflanzliche Medikamente (24) und Drogen (25) muss gedacht werden.

\section{Klinik des akuten Leberversagens}

Symptome und Beschwerden eines Patienten mit akutem Leberversagen setzen sich aus Zeichen der Leberschädigung zusammen, die gelegentlich durch Symptome der zu Grunde liegenden Erkrankung ergänzt werden können. Hepatische Encephalopathie und Ikterus gehören definitionsgemäß zum akuten Leberversagen $(5,6)$. Die weiter unten aufgeführten extrahepatischen Komplikationen (Abb. 2) treten erst im Verlauf der Erkrankung auf und sind bei Einweisung des Patienten nie vollständig vorhanden. Wei-

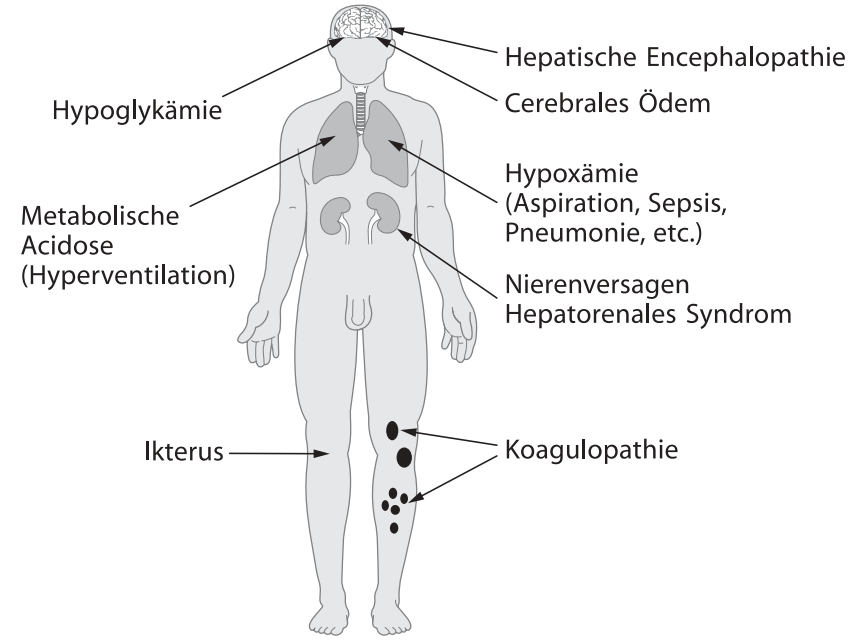

Abb. 2 Extrahepatische Komplikationen beim akuten Leberversagen

tere unspezifische klinische Befunde (tastbare Leber von vermehrter Konsistenz, Splenomegalie, LeberHaut-Zeichen etc.) und Symptome (Übelkeit, abdominelle Schmerzen, Juckreiz, dunkler Urin etc.) eines Leberleidens sind häufig vorhanden. Ätiologisch verwertbare Symptome sind selten. Dazu gehört zum Beispiel der Nachweis eines Kayser-FleischerschenKornealrings, der spezifisch für einen Morbus Wilson ist. Die seltene erythropoetische Protoporphyrie geht mit einer Schädigung peripherer Nerven und bullösen Hautveränderungen einher (26). Eigenoder Fremdanamnese sind hilfreich bei der Klärung einer viralen, medikamentösen oder toxinbedingten Genese des akuten Leberversagens. Die Bestimmung der Transaminasen erlaubt weder eine ätiologische Zuordnung noch eine Schweregradbeurteilung der Erkrankung.

\section{Komplikationen des akuten Leberversagens}

\section{Hepatische Enzephalopathie}

Das Auftreten einer hepatischen Enzephalopathie gehört definitionsgemäß zum akuten Leberversagen. Der pathogenetische Zusammenhang zwischen hepatischer Enzephalopathie und zerebralem Ödem ist ungeklärt. Es gibt Hinweise darauf, dass Ammoniak eine wichtige Rolle bei der Entstehung des Hirnödems und einer nachfolgenden tödlichen Einklemmung des Hirnstamms spielt $(27,28)$. Beide Syndrome gehen fließend ineinander über und haben nicht nur eine unterschiedliche Prognose, sondern bedürfen jeweils auch einer spezifischen Therapie (1). 
Beim akuten Leberversagen äußert sich die hepatische Enzephalopathie gelegentlich mit psychotischen Episoden, psychomotorischer Agitation, Krämpfen oder tiefem Koma, Infektionen, Elektrolytstörungen, Obstipation und Medikamente (Diuretika, Sedativa) begünstigen ähnlich wie beim chronischen Leberversagen die Entstehung einer hepatischen Enzephalopathie. Die auslösenden Faktoren müssen stets gesucht und gegebenenfalls therapeutisch angegangen werden. Die hepatische Enzephalopathie spricht beim akuten Leberversagen schlecht auf die Gabe von Laktulose (bis zu $3 \times 50 \mathrm{ml} \mathrm{p.o./Tag} \mathrm{über} \mathrm{eine} \mathrm{Magenson-}$ de) an. Zusätzlich können zu Beginn der Erkrankung bis $\mathrm{zu} 3 \times$ täglich Laktuloseeinläufe (300 ml Laktulose $+700 \mathrm{ml} \mathrm{H}_{2} \mathrm{O}$ ) durchgeführt werden. Bei ausgeprägter Koagulopathie muss hierauf jedoch verzichtet werden.

Bei einer Untergruppe von Patienten mit Koma bei dekompensierter Leberzirrhose führte die intravenöse Gabe von Flumazenil zu einer Besserung der hepatischen Enzephalopathie (29). Beim fulminanten Leberversagen wurde diese Substanz bisher noch nicht evaluiert. Schwer resorbierbare Antibiotika (Neomycin, Paromomycin) können, falls auf eine selektive Darmdekontamination verzichtet wird, hilfreich sein. Sie sollten jedoch nur zeitlich limitiert über bis zu einer Woche zum Einsatz kommen, da sie in geringem Ausmaß resorbiert werden und nephrotoxisch sind. Bisher noch experimentell eingesetzte Systeme zur extrakorporalen Blutentgiftung können zukünftig bei hepatischer Encephalopathie und Leberversagen möglicherweise hilfreich sein (30, $31)$.

\section{Hirnödem}

Ein zerebrales Ödem entwickelt sich bei $75-80 \%$ der Patienten mit akutem Leberversagen und einer hepatischen Enzephalopathie vom Schweregrad IV. Dagegen findet es sich nur bei $9 \%$ der Fälle mit chronischem Leberversagen.

Bei 20\% der Patienten mit zerebralem Ödem führt diese Komplikation über eine Hirnstammeinklemmung zum Tode. Es ist die häufigste Todesursache des akuten Leberversagens (32). Klinisch äußert sich das zerebrale Ödem in arterieller Hypertonie, Krämpfen, Tachykardie, Hyperventilation und/oder pathologischen Pupillen- und Hirnstammreflexen, aber nur selten in einem Papillenödem. $\mathrm{Zu}$ Beginn kann die Symptomatik paroxysmal auftreten.

Eine gleichzeitig bestehende hepatische Enzephalopathie oder intensivmedizinische Maßnahmen (Sedierung, Relaxation, Intubation) können die klinische Symptomatik maskieren, weshalb bei HE-Stadium III oder beim Übergang vom Stadium III zu IV eine Überwachung des intrazerebralen Drucks (ICP) (33) mittels einer epiduralen Drucksonde durchgeführt wird. Der epidurale Druckaufnehmer wird neurochirurgisch durch ein Bohrloch in der Schädelkalotte angebracht. Wenn auch das Überleben der Patienten mit fulminantem Leberversagen durch diese Maßnahme nicht beeinflusst wurde, so können aus den Druckwerten jedoch prognostische Aussagen abgeleitet und die Indikation $\mathrm{zu}$ drucksenkenden Maßnahmen gestellt werden (33). Die Computertomographie ist nicht ausreichend sensitiv, um den ICP einzuschätzen.

Ein erhöhter intrazerebraler Druck bei zerebralem Ödem muss behandelt werden, um die drohende Hirnstammeinklemmung oder eine Abnahme der zerebralen Durchblutung zu verhindern. Insbesondere sollten Maßnahmen vermieden werden, die einen Anstieg des ICP bewirken. Hierzu gehören PEEP-Beatmung, intratracheale Sekretabsaugung, Hämofiltration, Lagerungsmaßnahmen, Fieber, Husten, eine Hypoxämie oder Hyperkapnie (1). Der Wert einer prophylaktischen Absenkung des $\mathrm{pCO}_{2}$ auf $25-30 \mathrm{mmHg}$ durch Hyperventilation ist nicht erwiesen. Wird die $\mathrm{pCO}_{2}$-Absenkung $\mathrm{zu}$ forciert betrieben, kann hieraus eine Vasokonstriktion mit Abnahme der zerebralen Durchblutung resultieren.

Bei einem über mehrere Minuten anhaltenden Anstieg des ICP über $25 \mathrm{mmHg}$ wird Mannitol in einer Dosierung von $0,5-1,0 \mathrm{~g} / \mathrm{kg}$ Körpergewicht über $5 \mathrm{Mi}$ nuten verabreicht. Die Mannitol-Gabe kann stündlich wiederholt werden. Alternativ kann bei Nichtansprechen oder Versagen der Mannitolbehandlung Thiopental oder Pentobarbital intravenös injiziert werden (1). Ein Absenken der Kerntemperatur auf $32-33^{\circ} \mathrm{C}$ unter Verwendung von Kühldecken führte zu einer signifikanten Zunahme des cerebralen Perfusionsdruckes bei Patienten mit akutem Leberversagen und mit nicht kontrollierbarem erhöhten intrakraniellen Druck (34). Das gleiche Phänomen wurde nach extrakorporaler Albumindialyse bei 8 Patienten mit akutem Leberversagen beobachtet (35). Beide Methoden bedürfen für diese Indikation einer weiteren klinischen Evaluierung.

\section{Hypoglykämie}

Der Ausfall der Leberzellfunktion führt zu einer gestörten Insulinclearance mit erhöhten Insulinspiegeln und einer gestörten Glukoneogenese und Glykogenolyse (1). Hieraus resultieren häufige schwere hypoglykämische Episoden, die insbesondere in der Frühphase des Leberversagens 2-stündliche Bestimmungen der Blutglukose erforderlich machen. Unter Verwendung hochkonzentrierter Glukoselösungen (40\%) werden mindestens $300 \mathrm{~g}$ Glukose täglich verabreicht. 


\section{Infektionen}

80\% der Patienten mit akutem Leberversagen leiden an einer bakteriellen und über $30 \%$ an einer Pilzinfektion (36). Häufigste Ursachen für Infektionen beim akuten Leberversagen sind Pneumonien (47\%), Harnwegsinfektionen (23\%) und infizierte Katheter (4\%). Bei $30 \%$ der Patienten finden sich weder eine Leukozytose noch Fieber.

Bei Patienten ohne nachgewiesene Infektion reduzierte die prophylaktische Gabe von Antibiotika die Infektionshäufigkeit auf 20\% (36). Die Antibiotikaprophylaxe hatte jedoch keinen statistisch signifikanten Einfluss auf das Überleben oder die Dauer der Hospitalisierung. Insbesondere Patienten, bei denen keine Kontraindikation gegen eine Lebertransplantation besteht, profitieren von einer prophylaktischen Antibiotikatherapie, da unter diesen Bedingungen die Zahl und der Schweregrad septischer postoperativer Komplikationen abnehmen. Günstig ist hierbei die Kombination eines intravenösen BreitspektrumAntibiotikums mit einem oralen Antimykoticum. Die selektive Darmdekontamination mit schwer resorbierbaren oralen Antibiotika bringt keinen zusätzlichen Vorteil zu einer systemischen Antibiose.

\section{Kreislaufversagen}

Insbesondere bei gleichzeitig vorhandenem zerebralen Ödem ist die Aufrechterhaltung eines ausreichenden arteriellen Drucks mit Infusion von 5\%iger $\mathrm{Hu}-$ manalbuminlösung von vitaler Bedeutung. Bei $60 \%$ der Fälle mit akutem Leberversagen finden sich hämodynamische Verhältnisse wie bei einem septischen Zustand: Das Herzzeitvolumen ist erhöht, der periphere systemische Widerstand und der arterielle Blutdruck sind erniedrigt $(1,37)$.

Patienten mit Enzephalopathie Grad IV und entsprechend hohem Risiko, ein zerebrales Ödem zu entwickeln, müssen einen Pulmonaliskatheter erhalten, um eine adäquate Volumensubstitution durchzuführen und die peripheren Widerstände mit Katecholaminen präziser einstellen zu können. Die alleinige Volumenzufuhr ist häufig jedoch erfolglos, so dass die zusätzliche Gabe von Adrenalin oder Noradrenalin erforderlich wird. Dies führt zwar zu einer Zunahme des mittleren arteriellen Blutdrucks, lässt aber die Sauerstoffzufuhr nicht in gleicher Weise ansteigen. In einer kontrollierten Studie zeigte sich, dass N-Acetylcystein, das die Glutathionspeicher und möglicherweise die Stickoxid (NO)-Konzentration ansteigen lässt, und Prostazyklin, das zu einer Vasodilatation im Bereich der Mikrozirkulation führt, günstige Wirkungen auf Sauerstoffangebot und -verbrauch haben (38). In einer kürzlich publizierten
Studie wurde diese Beobachtung für das N-Acetylcystein jedoch in Frage gestellt (39). Der Austausch von 8-10 l Blutplasma gegen tiefgefrorenes Frischplasma bewirkte bei Patienten mit fulminantem Leberversagen eine Besserung des systemischen Gefäßwiderstandes und des arteriellen Blutdrucks $(40,41)$. Zukünftige Studien müssen zeigen, ob dieser therapeutische Ansatz oder ein extrakorporales Leberersatzverfahren zur Kreislaufstabilisierung beitragen können.

\section{Gerinnungsstörungen}

Aufgrund der Abnahme der funktionstüchtigen Leberzellmasse ist nicht nur die leberspezifische Synthese von Gerinnungsfaktoren sondern auch die von hepatischen Proteinaseinhibitoren, die in den Ablauf der Gerinnungskaskade eingreifen, deutlich herabgesetzt (42). Darüber hinaus kommt es zu einer gestörten hepatischen Clearance von aktivierten Gerinnungsfaktoren. Durch die vermehrte Freisetzung von Zytokinen und neutralen Proteinasen aus extrahepatischen Geweben wird die intravasale Gerinnung gefördert mit nachfolgendem gesteigerten Verbrauch und gleichzeitiger Hyperfibrinolyse. Das Gerinnungssystem befindet sich zumeist auf niedrigem Niveau im Gleichgewicht, da nicht nur Gerinnungsfaktoren sondern auch deren Antagonisten abfallen.

Die Aktivierung des fibrinolytischen Systems beim akuten Leberversagen spiegelt sich in der vermehrten Freisetzung von Plasmin und erhöhten Konzentrationen an Plasminogenaktivatoren wider. Antifibrinolytisch wirksame Substanzen führten jedoch $\mathrm{zu}$ einem weniger ausgeprägten Abfall, so dass dadurch die Blutungshäufigkeit durch Hyperfibrinolyse begrenzt werden kann (42). Die Daten hierzu sind jedoch nicht ausreichend, um einen Einsatz beim akuten Leberversagen zu empfehlen.

Trotz der stets vorhandenen Koagulopathie sollte die Substitution von Gerinnungsfaktoren restriktiv gehandhabt werden. Einmal enthalten diese Präparate gelegentlich aktivierte Gerinnungsfaktoren, die eine disseminierte intravasale Koagulopathie auslösen können, zum andern sind Faktor V (Halbwertzeit bis $\mathrm{zu}$ 1,5 Tagen) und der Quick-Wert wichtige prognostische Parameter, die nach Substitution nicht mehr verwertbar sind. Lediglich bei schwerer Gerinnungsstörung und aktiver Blutung wird man sich zur Gabe von Frischplasma (FFP) entscheiden (42). In kontrollierten Studien zeigte die prophylaktische Gabe von FFP (42) oder AT III (43) keinen Effekt auf Mortalität oder Morbidität der Patienten.

Ursachen für die bei akutem Leberversagen häufig vorliegenden erniedrigten Thrombozytenzahlen sind die stets ablaufende disseminierte intravasale Gerin- 
nung, eine gestörte Neubildung im Knochenmark durch toxische Substanzen oder Alkohol und ein vermehrter Abbau bei Hyperspleniesyndrom. Darüber hinaus scheinen verschiedene hepatozytenspezifische Faktoren (Thrombopoetin) für die Reifung der Blutplättchen und deren Freisetzung ins periphere Blut erforderlich $\mathrm{zu}$ sein. Zusätzlich besteht zumeist eine Thrombozytopathie mit gestörter Thrombozytenaggregation aber erhaltener Adhäsion, deren Ursache noch nicht vollständig aufgeklärt ist. Thrombozytenkonzentrate sind nur bei Patienten mit schweren Blutungen indiziert (42). Eine prophylaktische Gabe ist vor invasiven Eingriffen indiziert, wenn die Zahl unter $50000 / \mu l$ liegt. Ohne nachgewiesene Blutung sollte eine Thrombozytensubstitution nicht begonnen werden, wenn die Thrombozyten über 20000-30000/ $\mu$ liegen.

\section{Nierenversagen}

In bis zu $75 \%$ der Fälle von akutem Leberversagens kommt es zu einem oligurischen Nierenversagen (unter $300 \mathrm{ml}$ Urin/24 h) $(1,37)$. Die dieser Störung zugrundeliegenden Ursachen sind vielfältig: Direkte toxische Nierenschädigung (Paracetamol), ein hepatorenales Syndrom, Tubulusnekrosen oder ein prärenales Nierenversagen bei Hypotonie und Hypovolämie. Die Nierenretentionswerte geben beim Leberversagen nicht immer eine zuverlässige Aussage über das Ausmaß der Schädigung. In dieser Situation wird Harnstoff in der Leber vermindert synthetisiert und Kreatinin weniger aus der Peripherie mobilisiert.

Prinzipiell sollten nephrotoxische Medikamente wie Aminoglykoside, Prostaglandin-syntheseinhibitoren (nichtsteroidale Antirheumatika) oder auch Mannitol bei fulminantem Leberversagen nicht oder nur mit Vorsicht eingesetzt werden. Wichtig ist die Aufrechterhaltung eines ausreichenden intravasalen Volumens (ZVD von $10-12 \mathrm{~cm} \mathrm{H}_{2} \mathrm{O}$ ), wobei Dopamin in niedriger Dosis $(2-4 \mu \mathrm{g} / \mathrm{kg} / \mathrm{h})$ in Frühstadien fraglich eine Besserung des renalen Blutflusses und damit auch der Nierenfunktion bewirken kann.

Bei Vorliegen eines hepatorenalem Syndroms kann vorübergehend bis zur definitiven Therapie des Leberversagens durch eine Lebertransplantation die Gabe von Vasopressinanaloga (6 U/h als Dauerinfusion) von Nutzen sein (44). Die Wirkung von Ornipressin oder Terlipressin wird auf eine Verminderung der peripheren Vasodilatation mit Zunahme der renalen Perfusion zurückgeführt. Eine extrakorporale Albumindialyse (45) soll über die Entfernung von an Albumin gebundenen Substanzen günstige Auswirkungen auf das hepatorenale Syndrom haben. Diese Mitteilung bedarf aber der Bestätigung.

Eine schwere metabolische Azidose, Hyperkaliämie oder Volumenüberladung macht die Durchfüh- rung einer Hämodialyse erforderlich (37). Steigt unter Mannitoltherapie des zerebralen Ödems die Osmolalität $\mathrm{zu}$ stark an oder steigt das Kreatinin über $4,6 \mathrm{mg} / \mathrm{dl}(400 \mu \mathrm{mol} / \mathrm{l})$ ist ebenfalls die Indikation zur Hämodialyse gegeben. Bei Patienten mit bereits erhöhtem intrakraniellen Druck wird eine kontinuierliche veno-venöse oder arterio-venöse Hämofiltration eingesetzt (46). Bei diesen Verfahren ist auch von Vorteil, dass auf die Gabe von Heparin verzichtet werden kann. Die zu rasche Entfernung von osmotisch wirksamen Substanzen aus der Blutbahn kann ein zerebrales Ödem verstärken.

\section{Spezifische Therapie}

\section{Ätiologisch definierte Therapien}

Beim fulminanten Leberversagen steht eine weitgehend ätiologisch definierte Therapie bei Intoxikation mit Paracetamol oder dem Knollenblätterpilz und bei fulminanter CMV- oder HSV-Infektion zur Verfügung (Tab. 2). Hohe Dosen von N-Acetylcystein wirken antioxidativ und als Radikalenfänger und reduzierten auch bei verzögerter Applikation 10-36 Std. nach Paracetamolintoxikation (10-20 g) die Mortalität des akuten Leberversagens $(48,49)$. Je früher die Infusion von $\mathrm{N}$-Acetylcystein erfolgte, umso besser war die Prognose (22).

Die Behandlung der Knollenblätterpilzintoxikation (50) basiert nicht auf kontrollierten Studien. Empirisch werden neben Maßnahmen zur Verringerung der Giftaufnahme Silibinin, Steroide und die forcierte Diurese eingesetzt (Tab.2). Falls die Einnahme

Tab. 2 Ätiologisch definierte konservative Therapieansätze beim akuten Leberversagen

\begin{tabular}{|c|c|}
\hline Ursache des Leberversagens & Behandlung \\
\hline Paracetamol-Vergiftung & $\begin{array}{l}\text { N-Acetylcystein } 150 \mathrm{mg} / \mathrm{kg} / 15 \mathrm{Min} \text {. in } \\
250 \mathrm{ml} \text { Glucose } 5 \% \\
50 \mathrm{mg} / \mathrm{kg} / 4 \text { Std. in } 500 \mathrm{ml} \text { Glucose } 5 \% \\
100 \mathrm{mg} / \mathrm{kg} / 16 \mathrm{Std} \text {. in } 1000 \mathrm{ml} \text { Glucose } 5 \%\end{array}$ \\
\hline Knollenblätterpilz-Vergiftung & $\begin{array}{l}\text { Provokation von Erbrechen, Kohle- } \\
\text { applikation, abführende Maßnahmen, } \\
\text { intestinale Lavage } \\
\text { Penicillin G } 1 \text { Mio Einheiten } / \mathrm{kg} / \mathrm{Tag} \\
\text { Silibinin } 20 \mathrm{mg} / \mathrm{kg} / \mathrm{Tag} \text { (verteilt auf } \\
4 \text { Infusionen über jeweils } 3 \text { Stunden } \\
\text { pro Tag) }\end{array}$ \\
\hline Herpes simplex & Aciclovir \\
\hline Cytomegalievirus & Ganciclovir \\
\hline Budd-Chiari-Syndrom & TIPS oder Shunt-Operation \\
\hline HELLP-Syndrom & Beendigung der Schwangerschaft \\
\hline
\end{tabular}


des Pilzes nicht in suizidaler Absicht bei schwerer psychiatrischer Erkrankung erfolgte, ist auch in diesen Fällen rechtzeitig an eine Lebertransplantation zu denken.

Bei Schwangerschaft assoziiertem Leberversagen (akute Schwangerschaftsfettleber, HELLP-Syndrom) führt eine rechtzeitige Beendigung der Schwangerschaft in der Regel zu einer raschen Besserung und verhindert die Entstehung eines komplett ausgebildeten akuten Leberversagens.

\section{Pharmakologische Beeinflussung der Leberfunktion}

Zahlreiche Studien zeigten, dass regeneratorisch wirkende Substanzen nicht notwendigerweise einen günstigen Effekt beim akuten Leberversagen haben. Die Verabreichung von HGF (Hepatocyte growth factor) erwies sich tierexperimentell ohne Wirkung auf das durch Paracetamol induzierte Leberversagen (51). Unwirksam waren auch Glukagon, Insulin und $\alpha$-Interferon.

Prostaglandinen wurde eine hepatoprotektive Wirkung zugeschrieben. Prostaglandin $\mathrm{E}_{2}\left(\mathrm{PGE}_{2}\right)$ stimuliert unter anderem die Leberzellregeneration. In einer prospektiven, randomisierten und kontrollierten Studie an 41 Patienten mit akutem Leberversagen zeigte die Substanz keine Wirkung auf das Überleben, da $40 \%$ der Patienten unter $\mathrm{PGE}_{2}$ und $38 \%$ der Fälle in der Plazebogruppe überlebten (52). Besonders die Patienten mit frühem Behandlungsbeginn mit $\mathrm{PGE}_{2}$ zeigten jedoch einen deutlichen Trend $\mathrm{zu}$ einem verbesserten Überleben. Wurde die Therapie innerhalb von 10 Tagen nach Beginn des fulminanten Leberversagens aufgenommen, lag der Prozentsatz der Überlebenden bei $73 \%$ gegenüber $16 \%$ bei Therapiebeginn zu einem späteren Zeitpunkt. Derzeit ist keine dieser Substanzen für einen Einsatz in der Routine geeignet.

\section{Extrakorporaler Leberersatz}

Bisher ist noch nicht eindeutig geklärt, welche Leberfunktion für das Überleben des Patienten von entscheidender Bedeutung ist und somit vordringlich ersetzt werden sollte $(1,53)$. Verfahren, die nur partielle Leistungen der Leber ersetzen, haben sich bisher als wenig erfolgreich erwiesen. Nach Möglichkeit sollten sämtliche Leberfunktionen solange ersetzt werden, bis eine ausreichende Regeneration der geschädigten Leber stattgefunden hat. Hierzu wurden Hepatozytenkulturen oder Hepatomzellen eingesetzt, die weitgehend die Eigenschaften menschlicher Hepatozyten besitzen (53).

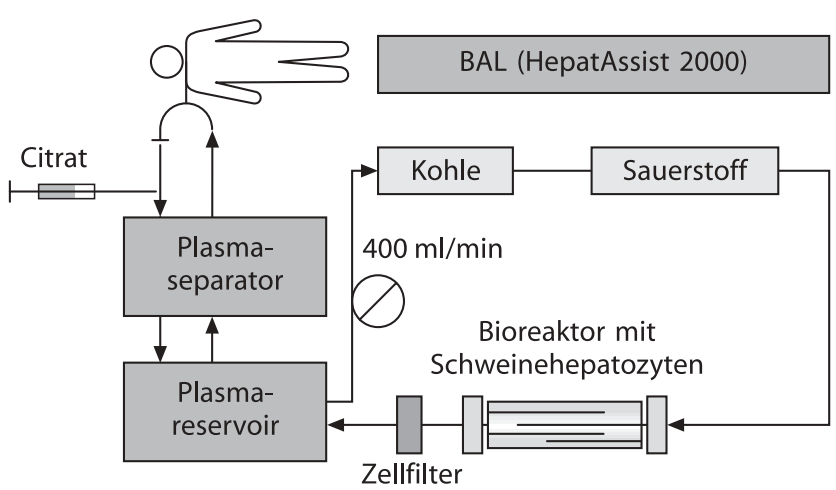

Abb. 3 Anschluss der künstlichen Leber (BAL-System, "bioartificial liver") an den Kreislauf des Patienten

In einem Prototypen einer künstlichen Leber befinden sich Schweinehepatozyten auf der Außenseite von hohlen, porösen Celluloseacetatfasern, die auf mit Kollagen beschichtetem, kugelförmigem Trägermaterial angewachsen sind (Dextran-Microcarrier). Vorgeschaltet ist eine Plasmapheresevorrichtung (Abb. 3). Das mit Citrat versetzte Plasma wird zunächst über einen Kohlefilter und danach im Bioreaktor über die Celluloseacetatfasern mit den Schweinehepatozyten in Kontakt gebracht. Von 18 Patienten mit akutem Leberversagen konnten in einer ersten Untersuchung 16 später erfolgreich lebertransplantiert werden (54). Weitere Studien müssen den tatsächlichen klinischen Wert dieser künstlichen Leber (BAL, bioartificial liver) prüfen.

Ein anderer extrakorporaler Bioreaktor (ELAD; Extracorporeal liver assist device) benutzt ebenfalls Hohlfasern, die zwischen hoch differenzierten menschlichen Hepatom-Zellen (C3a) verlaufen und venovenös perfundiert werden. In einer kontrollierten Studie an Patienten mit 50\% Überlebenschance $(\mathrm{n}=17)$ oder mit dringlicher Transplantationsindikation $(n=7)$, zeigte sich eine geringe Besserung von Leberfunktionsparametern, ohne dass dies jedoch einen signifikanten Effekt auf das Überleben hatte (55).

Die extrakorporale Xenoperfusion einer intakten Schweineleber führte zu vorübergehenden neurologischen und biochemischen Besserungen bei 3 Patienten, die jedoch im weiteren Verlauf an dem fulminanten Leberversagen starben (56). Ein vierter Patient konnte über 10 Tage bis zu einer erfolgreichen Lebertransplantation stabilisiert werden. Nicht nur tierische Lebern sondern auch humane Lebern, die für eine Transplantation ungeeignet erscheinen, können extrakorporal perfundiert werden. Auch hierzu gibt es einzelne Fallberichte.

Beim akuten Leberversagen sind eine Vielzahl von möglicherweise pathogenetisch bedeutsamen Meta- 
boliten an Albumin gebunden. Mittels Albumindialyse können diese Substanzen in einem zellfreien System aus dem Blut der Patienten entfernt werden. Bei der extrakorporalen Albumindialyse (MARS; molecular absorbent recirculating system) wird das Blut durch Hohlfasern geleitet und gegen 15\%ige Albuminlösung dialysiert (Molekulargewicht $<60 \mathrm{kd}$ ). Die am Albumin des Dialysates gebundenen Metaboliten werden kontinuierlich durch Überleitung über eine Kohlekartusche und einen Anionenaustauscher entfernt, so dass das eingesetzte Albumin kontinuierlich regeneriert wird. Zwar konnte in einer ersten kontrollierten Studie das 30-Tage-Überleben von Patienten mit Leberzirrhose und akutem Leberversagen signifikant verbessert werden, nach 3 Monaten war dieser Unterschied jedoch nicht mehr vorhanden (57). Kritikpunkte an dieser Studie sind zahlreich $(58,59)$, so dass ein Einsatz dieses Verfahrens außerhalb von Studien derzeit nicht empfohlen wird. Vorsicht impliziert auch die Beobachtung der Auslösung einer disseminierten intravasalen Gerinnung mit klinisch signifikanten Blutungen (60).

\section{Intrakorporaler Leberersatz}

Der intrakorporale Leberersatz kann drei unterschiedliche Ziele haben: a) Überbrückung der Zeit bis zur Verfügbarkeit einer neuen Leber und anschließender Transplantation, b) Überbrückung der Zeit bis zur Regeneration der eigenen Leber mit Rückbildung der implantierten auxiliären Leber oder Verschwinden der transplantierten Hepatozyten oder c) dauerhaftes Angehen und Funktionieren des Transplantats.

Eine der Möglichkeiten, intrakorporal vorübergehend die Leberfunktion zu ersetzen, besteht in der Transplantation von Hepatozyten (61). Dieses Verfahren kann möglicherweise sowohl einen größeren chirurgischen Eingriff wie die Lebertransplantation als auch eine lebenslange Immunsuppression vermeiden. Die intraportale oder intralienale Infusion der Zellen bei akutem Leberversagen führte sowohl zur Reduktion der Ammoniakkonzentration im Serum als auch des intrazerebralen Drucks (61). Trotz dieser ersten positiven Beobachtungen am Menschen gibt es noch viele Probleme mit diesem Verfahren: Die Zellen benötigen Tage nach Transplantation bis sie ihre leberspezifischen Funktionen wieder erlangt haben; das toxische Agens, das zum Leberversagen geführt hat, schädigt auch die infundierten Hepatozyten; schließlich ist auch die Zahl der transplantierbaren Leberzellen und ihre rasche Verfügbarkeit limitiert. Somit ist dieses Verfahren derzeit noch als experimentell $\mathrm{zu}$ betrachten.

Die auxiliäre Lebertransplantation ist ein weiteres invasives Verfahren um die Zeit bis zur Regeneration der eigenen geschädigten Leber zu überbrücken. Bei der auxiliären heterotopen Lebertransplantation wird die Spenderleber in den rechten oberen abdominellen Quadranten implantiert, während bei orthotoper auxiliärer Transplantation die eigene Leber teilweise reseziert und durch eine Hälfte einer Spenderleber ersetzt wird. In einer europäischen Multicenterstudie überlebten 29 von 47 Patienten mit akutem Leberversagen nach auxiliärer Lebertransplantation (62). Das UUberleben der Patienten und die Häufigkeit einer Retransplantation war nicht verschieden zwischen komplettem orthotopen Leberersatz und auxiliärer Transplantation. Die orthotope Transplantation der auxiliären Leber erwies sich mit 71\% 1-Jahresüberleben gegenüber der heterotopen Transplantation mit $33 \%$ als überlegen. $2 / 3$ der Überlebenden waren nach einem Jahr ohne Immunsuppression. Falls im Einzelfall präzisere Aussagen zur Regenerationsfähigkeit der Leber gemacht werden können, wird dieses Verfahren die Standardtherapie des akuten Leberversagens werden. Wahrscheinlich profitieren insbesondere junge Patienten und solche mit viralen Hepatitiden und einem raschen Beginn des fulminanten Leberversagens.

Die permanente orthotope Lebertransplantation (63) ist ein etabliertes Verfahren zur Behandlung des akuten Leberversagens mit Überlebensraten von $60-70 \%$ (1). Aus diesem Grunde sollten frühzeitig an eine Lebertransplantation gedacht und Kontraindikationen ausgeschlossen werden (Tab. 3) (64). Viele Patienten versterben während sie auf ein Spenderorgan warten. Wenn eine alleinige konservative Therapie die Überlebenschancen auf unter $20 \%$ sinken lässt, ist die Indikation für eine Lebertransplantation gegeben. Die rechtzeitige Verlegung dieser Patienten in ein Transplantationszentrum ist für den weiteren Verlauf von entscheidender Bedeutung. Auch beim akuten Leberversagen wurde die Transplantation von Teillebern als Lebendspende bereits erfolgreich eingesetzt (65).

Tab. 3 Kontraindikationen für eine Lebertransplantation beim fulminanten Leberversagen

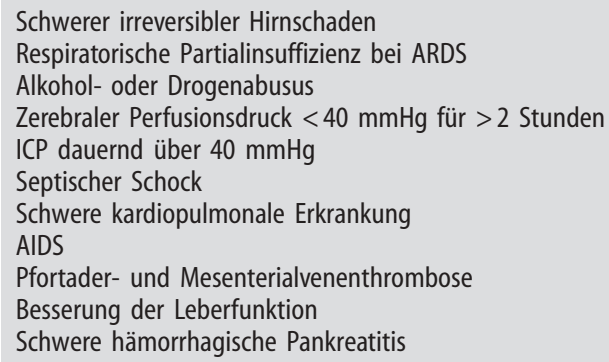




\section{Prognose des akuten Leberversagens}

Für die schwierige Entscheidung, wann der Zeitpunkt für eine Lebertransplantation bei einem akuten Leberversagen gekommen ist, stehen verschiedene prognostische Parameter zur Einschätzung der Überlebenswahrscheinlichkeit der Patienten zur Verfügung (1). Die Prognose wird entscheidend von der Ätiologie der Lebererkrankung bestimmt. Patienten mit einer Hepatitis A, Hepatitis B oder Paracetamolintoxikation haben eine günstigere Prognose als die mit einer NANB-Hepatitis oder Medikamenten- bzw. Halothanschädigung. Die Prognose wird weiterhin von demographischen (Alter) und biochemischen Parametern (Lebervolumen (66), Bilirubin, Gerinnungswerte, pH-Wert, Laktat (67) oder Phosphat (68) im Serum) beeinflusst.

Einige dieser Parameter gehen in die beiden besten Modelle zur Prognosebeurteilung in unterschiedlicher Weise ein: in die "Clichy“- und die „King'sCollege"-Kriterien. Die Clichy-Kriterien $(1,69)$ setzen eine hepatische Encephalopathie voraus. Weiterhin enthalten diese Kriterien das Alter und die Faktor V-Konzentration (Tab. 4). 95\% der Patienten mit akutem Leberversagen überlebten, wenn diese pathologischen Parameter nicht erfüllt waren. Waren die Bedingungen für eine schlechte Prognose erfüllt, so überlebten nur $10 \%$ ohne Lebertransplantation, aber $84 \%$ mit diesem Eingriff.

Die King's-College-Kriterien $(1,70,71)$ unterscheiden zwischen Patienten mit und ohne Paraceta-

Tab. 4 Prognostische Parameter beim akuten Leberversagen: Clichy-Kriterien

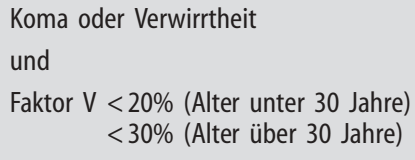

Tab. 5 Prognostische Parameter beim akuten Leberversagen: King's-CollegeKriterien

\begin{tabular}{ll}
\hline Paracetamolintoxikation & Andere Ursachen \\
\hline $\begin{array}{l}\mathrm{PH}<7,3 \\
\text { (unabhängig vom Schweregrad } \\
\text { der HE) }\end{array}$ & $\begin{array}{l}\text { Prothrombinzeit }<10 \% \\
\text { (unabhängig vom Schweregrad der } \\
\text { HE) }\end{array}$ \\
oder & oder (drei der folgenden Kriterien) \\
- Prothrombinzeit $<10 \%$ & - Alter $<10$ Jahre oder $>40$ Jahre \\
- Serumkreatinin $>3,4 \mathrm{mg} / \mathrm{dl}$ & - Ätiologie: NANB, Halothan, Medika- \\
& - Ikterusbeginn $>7$ Tage vor Beginn \\
- HE-Grad III oder IV & der HE \\
& - Prothrombinzeit $<20 \%$ \\
& - Serumbilirubin $>17,5 \mathrm{mg} / \mathrm{dl}$
\end{tabular}

molintoxikation (Tab. 5). Werden die Kriterien einer ungünstigen Prognose erfüllt, kann mit fast $90 \%$ Sicherheit angenommen werden, dass der Patient das Leberversagen bei alleiniger konservativer Therapie nicht überlebt. Waren dagegen die Kriterien nicht erfüllt, lag die Sicherheit, dass der Patient tatsächlich auch überlebte, bei $65 \%$. Die King's-College-Kriterien erwiesen sich zur Prognosebeurteilung der Paracetamolvergiftung als besser, während bei allen anderen Ursachen die Clichy-Kriterien gleichwertig sind und aufgrund ihrer einfacheren Handhabung Vorteile besitzen (69). Andere an der jeweiligen Ätiologie orientierte Scores sind komplizierter und weniger evaluiert.

Der bei massivem Leberzellzerfall messbare Abfall des im Blutplasma vorhandenen Gc-Proteins oder wiederholte Messungen von sensorisch evozierten Potentialen können möglicherweise zukünftig zur Prognoseeinschätzung beim akuten Leberversagen eingesetzt werden, wenn ihre Wertigkeit in weiteren klinischen Studien abgesichert wird.

\section{Zusammenfassung}

Die Verbesserung der Intensivmedizin in den letzten Jahren erlaubt eine bessere Überwachung und konservative Therapie der extrahepatischen Organkomplikationen beim akuten Leberversagen. Epidurale Drucksonden beeinflussen therapeutische Maßnahmen beim cerebralen Ödem, das durch Hirnstammeinklemmung und Tod kompliziert ist. Weitere prophylaktische Maßnahmen verhindern Komplikationen durch Hypoglykämie und bakterielle Infektionen. Neue Therapieoptionen können hilfreich beim Kreislauf- und Nierenversagen sein. Gut angelegte Studien zeigten weiterhin, dass beim Ersatz von Gerinnungsfaktoren Zurückhaltung eher von Vorteil ist.

Verschlechtert sich der Patient mit akutem Leberversagen, so muss eine Entscheidung für oder gegen eine Lebertransplantation aufgrund wiederholter täglicher Prognoseeinschätzungen unter Verwendung entsprechender Scores (Abb. 4) erfolgen (72). Dabei spielt auch die klinische Erfahrung eine ganz entscheidende Rolle. Die orthotope Lebertransplantation und auch die auxiliäre Lebertransplantation bieten entsprechend ausgewählten Patienten eine etwa $60 \%$ ige Überlebenschance. Bei den Fällen, für die kein Spenderorgan rechtzeitig zur Verfügung steht, ermöglichen Methoden des Leberersatzes, wie extrakorporale Albumin Dialyse, die extrakorporale Leberperfusion bzw. Perfusion von Zellkulturen oder die intracorporale Implantation von Hepatozyten, Zeit für die Regeneration der eigenen Leber oder die Bereitstellung einer Spenderleber zu gewinnen. Aller- 


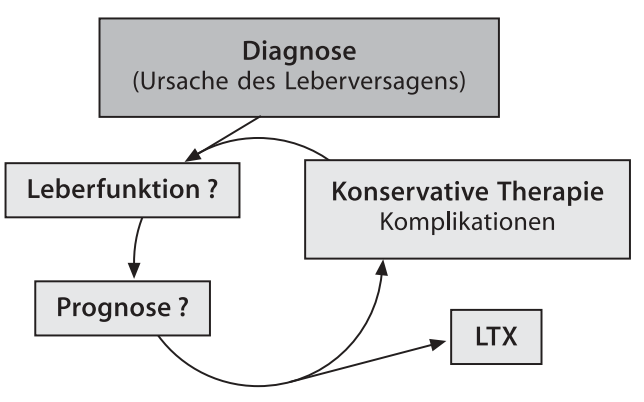

Abb. 4 Täglich mehrfach zu absolvierender diagnostischer Kreislauf zur rechtzeitigen Entscheidung für eine Lebertransplantation bei akutem Leberversagen

dings sind diese Optionen noch experimentell und bedürfen dringend der weiteren Evaluierung innerhalb kontrollierter klinischer Studien. Die auxiliäre Lebertransplantation stellt wahrscheinlich derzeit die beste Therapieoption für einen ausgewählten Patientenkreis dar, um vorübergehend die Leberfunktion aufrecht zu erhalten, bis die eigene Leber diese Aufgabe wieder übernimmt. Hierdurch wird eine lebenslange Abhängigkeit von Medikamenten und medizinischer Versorgung vermieden.

Versuche, die Regeneration der geschädigten Lebern mit Cytokinen und anderen Mediatoren $\mathrm{zu}$ stimulieren, sind bisher fehlgeschlagen. Die Prognose einiger aber nicht aller Patienten mit akutem Leberversagen hat sich in den letzten Jahren deutlich gebessert. Die besten Chancen haben wahrscheinlich die, die in einem Zentrum behandelt werden, an dem Hepatologen und Transplantationschirurgen eng zusammenarbeiten. Die beste Prophylaxe gegen ein akutes Leberversagen ist die von der ständigen Impfkommission empfohlene Impfung von Jugendlichen und Risikopersonen gegen das Hepatitis-B-Virus. Da eine HAV-Infektion bei Patienten mit chronischer Hepatitis $\mathrm{C}$ ein akutes Leberversagen auslösen kann, sollten diese Personen bei Auslandsreisen oder beruflicher Exposition entsprechend geimpft werden.

\section{Literatur}

1. Holstege A, Lock G, Köllinger M, Schölmerich J (1996) Conservative treatment of acute hepatic failure. Z Gastroenterol 34:192-201

2. Fagan EA (1999) Intrahepatic cholestasis of pregnancy. Clin Liver Dis 3: 603-632

3. Sallie R, Katsiyiannakis L, Baldwin D, Davies S, O'Grady J, Mowat A, MieliVergani G, Williams R (1992) Failure of simple biochemical indexes to reliably differentiate fulminant Wilson's disease from other causes of fulminant liver failure. Hepatology 12061211

4. Trey C, Davidson CS (1970) The management of fulminant hepatic failure. In: Popper H, Schaffner F (Hrsg) Progress in Liver Failure. Grune and Stratton, New York, S 292-298

5. O'Grady JG, Schalm SW, Williams R (1993) Acute liver failure: redefining the syndromes. Lancet 34:273-275

6. Tibbs C, Williams R (1995) Viral causes and management of acute liver failure. J Hepatol 22(suppl 1):68-73

7. Bernuau J, Benhamou JP (1993) Classifying acute liver failure. Lancet 342 : 252-253

8. Williams R (1996) Classification, etiology, and considerations of outcome in acute liver failure. Sem Liver Dis $16: 343-348$
9. Wu JC, Chen CL, Hou MC, Chen TZ, Lee SD, Lo KJ (1994) Multiple viral infection as the most common cause of fulminant and subfulminant viral hepatitis in an area endemic for hepatitis B: application and limitations of the polymerase chain reaction. Hepatology 19:836-840

10. O'Grady JG (1992) Management of acute and fulminant hepatitis A. Vaccine 10(suppl 1):S21-S23

11. Schiodt FV, Atillasoy E, Shakil AO, Schiff ER, Caldwell C, Kowdley KV, Stribling R, Crippin JS, Flamm S, Somberg KA, Rosen H, McCashland TM, Hay JE, Lee WM (1999) Etiology and outcome for 295 patients with acute liver failure in the United States. Liver Transpl Surg 5:29-34

12. Fagan EA, Harrison TJ (1994) Exclusion in liver by polymerase chain reaction of hepatitis B and C viruses in acute liver failure attributed to sporadic non-A, non-B hepatitis. J Hepatol 21:587-591

13. Carman WF, Thomas HC, Zuckerman AJ, Harrison TJ (1998) Molecular variants of hepatitis $B$ virus. In: Zuckerman AJ, Thomas HC (Hrsg) Viral Hepatitis, 2nd ed. Churchill Livingstone, London, S 141-172
14. Sterneck M, Kalinina T, Gunther S, Fischer L, Santantonio T, Greten H, Will H (1998) Functional analysis of HBV genomes from patients with fulminant hepatitis. Hepatology 28:13901397

15. Chu CM, Sheen IS, Liaw YF (1994) The role of hepatitis $\mathrm{C}$ virus in fulminant viral hepatitis in an area with endemic hepatitis A and B. Gastroenterology 107:189-195

16. Vento S, Garafano T, Renzini C, Cainelli F, Casali F, Ghironzi G, Ferraro T, Concia E (1998) Fulminant hepatitis associated with hepatitis A virus superinfection in patients with chronic hepatitis C. N Engl J Med 338:286-290

17. Vento S, Cainelli F, Mirandola F, Cosco L, Di Perri G, Solbiati M, Ferraro T, Concia E (1996) Fulminant hepatitis on withdrawal of chemotherapy in carriers of hepatitis C virus. Lancet 347:92-93

18. Acharya SK, Dasarathy S, Kumer TL, Sushma S, Prasanna KS, Tandon A, Sreenivas V, Nijhawan S, Panda SK, Nanda SK, Irshad M, Joshi YK, Duttagupta S, Tandon RK, Tandon BN (1996) Fulminant hepatitis in a tropical population: clinical course, cause, and early predictors of outcome. Hepatology 23:1448-1455 
19. Hamid SS, Jafri SM, Khan $\mathrm{H}$, Shah $\mathrm{H}$, Abbas Z, Fields H (1996) Fulminant hepatic failure in pregnant women: acute fatty liver or acute viral hepatitis? J Hepatol 25:20-27

20. Suzuki K, Aikawa T, Okamoto H (2002) Fulminant hepatitis $\mathrm{E}$ in Japan. N Engl J Med 347:1456

21. Rowbotham D, Wendon J, Williams R (1998) Acute liver failure secondary to hepatic infiltration: a single centre experience of 18 cases. Gut 42:576580

22. Schmidt LE, Dalhoff K, Poulsen HE (2002) Acute versus chronic alcohol consumption in acetaminophen-induced hepatotoxicity. Hepatology 35:876-882

23. Zimmerman HJ, Maddrey WC (1995) Acetaminophen (paracetamol) hepatotoxicity with regular intake of alcohol: analysis of instances of therapeutic misadventure. Hepatology 22:767773

24. Kraft M, Spahn TW, Menzel J, Senninger $\mathrm{N}$, Dietl $\mathrm{KH}$, Herbst $\mathrm{H}$, Domschke W, Lerch MM (2001) Fulminantes Leberversagen nach Einnahme des pflanzlichen Antidepressivums Kava-Kava. Dtsch Med Wschr 126:970-972

25. Ellis AJ, Wendon JA, Portmann B, Williams R (1996) Acute liver damage and ecstasy ingestion. Gut 38:454-458

26. Lock G, Holstege A, Mueller AR, Christe W, Doss MO, Scholmerich J, Neuhaus P (1996) Liver failure in erythropoietic protoporphyria associated with choledocholithiasis and severe post-transplantation polyneuropathy. Liver 16:211-217

27. Clemmesen JO, Larsen FS, Kondrup J, Hansen BA, Ott P (1999) Cerebral herniation in patients with acute liver failure is correlated with arterial ammonia concentration. Hepatology 29: 648-653

28. Strauss GI, Knudsen GM, Kondrup J, Moller K, Larsen FS (2001) Cerebral metabolism of ammonia and amino acids in patients with fulminant hepatic failure. Gastroenterology 121: 1109-1119

29. Gyr K, Meier R, Haussler J, Bouletreau P, Fleig WE, Gatta A, Holstege A, Pomier-Layrargues G, Schalm SW, Groeneweg M, Scollo-Lavizzari G, Ventura E, Zeneroli ML, Williams R, Yoo Y, Amrein R (1996) Evaluation of the efficacy and safety of flumazenil in the treatment of portal systemic encephalopathy: a double blind, randomised, placebo controlled multicentre study. Gut 39:319-324

30. Ash SR (2002) Extracorporeal blood detoxification by sorbents in treatment of hepatic encephalopathy. Adv Ren Replace Ther 9:3-18
31. Schmidt LE, Svendsen LB, Sorensen VR, Hansen BA, Larsen FS (2001) Cerebral blood flow velocity increases during a single treatment with the molecular adsorbents recirculating system in patients with acute on chronic liver failure. Liver Transpl 7:709-712

32. Ferenci P (1994) Brain dysfunction in fulminant hepatic failure. J Hepatol 21:487-490

33. Donovan JP, Schafer DF, Shaw BW Jr, Sorrell MF (1998) Cerebral oedema and increased intracranial pressure in chronic liver disease. Lancet 351:719721

34. Jalan R, Damink SW, Deutz NE, Lee A, Hayes PC (1999) Moderate hypothermia for uncontrolled intracranial hypertension in acute liver failure. Lancet 354:1164-1168

35. Schmidt LE, Svendsen LB, Sorensen VR, Hansen BA, Larsen FS (2001) Cerebral blood flow velocity increases during a single treatment with the molecular adsorbents recirculating system in patients with acute on chronic liver failure. Liver Transpl 7:709-712

36. Rolando N, Philpott-Howard J, Williams R (1996) Bacterial and fungal infection in acute liver failure. Semin Liver Dis 16:389-402

37. Ellis A, Wendon J (1996) Circulatory, respiratory, cerebral, and renal derangements in acute liver failure: pathophysiology and management. Sem Liver Dis 16:379-388

38. Harrison PM, Wendon JA, Gimson AE, Alexander GJ, Williams R (1991) Improvement by acetylcysteine of hemodynamics and oxygen transport in fulminant hepatic failure. N Engl J Med 324:1852-1857

39. Walsh TS, Hopton P, Philips BJ, Mackenzie SJ, Lee A (1998) The effect of $\mathrm{N}$-acetylcysteine on oxygen transport and uptake in patients with fulminant hepatic failure. Hepatology 27:1332-1340

40. Clemmesen JO, Larsen FS, Ejlersen E, Schiodt FV, Ott P, Hansen BA (1997) Haemodynamic changes after highvolume plasmapheresis in patients with chronic and acute liver failure. Eur J Gastroenterol Hepatol 9:55-60

41. Clemmesen JO, Gerbes AL, Gulberg V, Hansen BA, Larsen FS, Skak C, Tygstrup N, Ott P (1999) Hepatic blood flow and splanchnic oxygen consumption in patients with liver failure. Effect of high-volume plasmapheresis. Hepatology 29:347-355

42. Pereira SP, Langley PG, Williams R (1996) The management of abnormalities of hemostasis in acute liver failure. Sem Liver Dis 16:403-414
43. Langley PG, Hughes RD, Forbes A, Keays R, Williams R (1993) Controlled trial of antithrombin III supplementation in fulminant hepatic failure. J Hepatol 17:326-331

44. Guevara M, Gines P, FernandezEsparrach G, Sort P, Salmeron JM, Jimenez W, Arroyo V, Rodes J (1998) Reversibility of hepatorenal syndrome by prolonged administration of ornipressin and plasma volume expansion. Hepatology 27:35-41

45. Mitzner SR, Stange J, Klammt S, Risler T, Erley CM, Bader BD, Berger ED, Lauchart W, Peszynski P, Freytag J, Hickstein H, Loock J, Lohr JM, Liebe S, Emmrich J, Korten G, Schmidt R (2000) Improvement of hepatorenal syndrome with extracorporeal albumin dialysis MARS: results of a prospective, randomized, controlled clinical trial. Liver Transpl 6:277-286

46. Davenport A, Will EJ, Davison AM, Swindells S, Cohen AT, Miloszewski KJ, Losowsky MS (1989) Changes in intracranial pressure during haemofiltration in oliguric patients with grade IV hepatic encephalopathy. Nephron 53:142-146

47. Hoofnagle JH, Carithers RL Jr, Shapiro C, Ascher N (1995) Fulminant hepatic failure: summary of a workshop. Hepatology 21:240-252

48. Vale JA, Proudfoot AT (1995) Paracetamol (acetaminophen) poisoning. Lancet 346:547-552

49. Keays R, Harrison PM, Wendon JA, Forbes A, Gove C, Alexander GJ, Williams R (1991) Intravenous acetylcysteine in paracetamol induced fulminant hepatic failure: a prospective controlled trial. Brit Med J 303:10121019

50. Molling J, Pohle W, Klein H, Welte T (1995) Stellenwert therapeutischer Maßnahmen bei Vergiftung mit dem Knollenblätterpilz. Intensivmed 32: 37-42

51. Francavilla A, Azzarone A, Carrieri G, Cillo U, Van Thiel D, Subbottin V, Starzl TE (1993) Administration of hepatic stimulatory substance alone or with other liver growth factors does not ameliorate acetaminopheninduced liver failure. Hepatology 17:429-433

52. Sheiner P, Sinclair S, Greig P, Logan A, Blendis LM, Levy G (1992) A randomized controlled trial of prostaglandin $\mathrm{E}_{2}\left(\mathrm{PGE}_{2}\right)$ in the treatment of fulminant hepatic failure. Hepatology $16: 88 \mathrm{~A}$

53. Allen JW, Hassanein T, Bhatia SN (2001) Advances in bioartificial liver devices. Hepatology 34:447-455 
54. Watanabe FD, Mullon CJ-P, Hewitt WR, Arkadopoulos N, Kahaku E, Eguchi S, Khalili T, Arnaout W, Shackleton CR, Rozga J, Solomon B, Demetriou AA (1997) Clinical experience with a bioartificial liver in the treatment of severe liver failure. A phase I clinical trial. Ann Surg 225:484-494

55. Ellis AJ, Hughes RD, Wendon JA, Dunne J, Langley PG, Kelly JH, Gislason GT, Sussman NL, Williams R (1996) Pilot-controlled trial of the extracorporeal liver assist device in acute liver failure. Hepatology 24: 1446-1451

56. Chari RS, Collins BH, Magee JC, DiMaio JM, Kirk AD, Harland RC, McCann RL, Platt JL, Meyers WC (1994) Brief report: treatment of hepatic failure with ex vivo pig-liver perfusion followed by liver transplantation. N Engl J Med 331:234-237

57. Heemann U, Treichel U, Loock J, Philipp T, Gerken G, Malago M, Klammt S, Loehr M, Liebe S, Mitzner S, Schmidt R, Stange J (2002) Albumin dialysis in cirrhosis with superimposed acute liver injury: a prospective, controlled study. Hepatology 36 : 949-958

58. Sen S, Jalan R, Williams R (2002) Extracorporeal albumin dialysis in acute-on-chronic liver failure: will it stand the test of time? Hepatology 36:1014-1016

59. Kamath PS (2002) Is there life in MARS? Hepatology 36:1017-1019
60. Mullhaupt B, Kullak-Ublick GA, Ambuhl P, Maggiorini M, Stocker R, Kadry Z, Clavien PA, Renner EL (2002) First clinical experience with Molecular Adsorbent Recirculating System (MARS) in six patients with severe acute on chronic liver failure. Liver 22(Suppl 2):59-62

61. Soriano HE (2002) Liver cell transplantation: human applications in adults and children. In: Gupta S, Jansen PLM, Klempnauer J, Manns MP (ed) Hepatocyte Transplantation. Kluwer Academic Publishers, Dordrecht, S 99-115

62. van Hoek B, de Boer J, Boudjema $\mathrm{K}$, Williams R, Corsmit O, Trepstra OT (1999) Auxiliary versus orthotopic liver transplantation for acute liver failure. EURALT Study Group. European Auxiliary Liver Transplant Registry. J Hepatol 30:699-705

63. Bismuth H, Samuel D, Castaing D, Williams R, Pereira SP (1996) Liver transplantation in Europe for patients with acute liver failure. Sem Liver Dis 16:415-425

64. Holstege A (2002) Indikationen zur Lebertransplantation beim chronischen Leberversagen. Z Gastroenterol 40:891-902

65. Sterneck M, Fischer L, Buggisch P, Malago M, Rogiers X, Burdelski M, Greten H, Broelsch CE (1996) Transplantation of complete and split liver grafts for patients with fulminant hepatic failure. $\mathrm{Z}$ Gastroenterol 34: 795-800
66. Sekiyama K, Yoshiba M, Inoue K, Sugata F (1994) Prognostic value of hepatic volumetry in fulminant hepatic failure. Dig Dis Sci 39:240-244

67. Bernal W, Donaldson N, Wyncoll D, Wendon J (2002) Blood lactate as an early predictor of outcome in paracetamol-induced acute liver failure: a cohort study. Lancet 359:558-563

68. Schmidt LE, Dalhoff K (2002) Serum phosphate is an early predictor of outcome in severe acetaminophen-induced hepatotoxicity. Hepatology 36: 659-665

69. Izumi S, Langley PG, Wendon J, Ellis AJ, Pernambuco RB, Hughes RD, Williams R (1996) Coagulation factor V levels as a prognostic indicator in fulminant hepatic failure. Hepatology 23:1507-1511

70. Anand AC, Nightingale P, Neuberger JM (1997) Early indicators of prognosis in fulminant hepatic failure: an assessment of the King's criteria. J Hepatol 26:62-68

71. Pauwels A, Mostefa-Kara N, Florent C, Levy VG (1993) Emergency liver transplantation for acute liver failure. Evaluation of London and Clichy criteria. J Hepatol 17:124-127

72. Zimmerman JE, Wagner DP, Seneff MG, Becker RB, Sun X, Knaus WA (1996) Intensive care unit admissions with cirrhosis: risk-stratifying patient groups and predicting individual survival. Hepatology 23:1393-1401 